\title{
Schweiz: dritthöchste Gesundheitsausgaben im neuesten OECD-Vergleich
}

\author{
Auf drei verschiedenen Ranglisten gemäss neuester OECD-CD hatte die Schweiz \\ 2007 das drittteuerste Gesundheitssystem der 30 OECD-Länder. Unsere nationalen \\ Gesundheitsausgaben entsprechen $10,3 \%$ des Bruttoinlandprodukts BIP. Dieser \\ fünfte SÄZ-Artikel zu den OECD-Jahresstatistiken enthält erstmals Daten über Arzt- \\ besuche, Präventionsausgaben und durchschnittliche Bettenbelegung. Zum zweiten \\ Mal nach 2006 thematisiert ist die Medizintechnik.
}

Gerhard Kocher

Korrespondenz:

Dr. rer. pol. Gerhard Kocher Haldenweg $10 \mathrm{~A}$

$\mathrm{CH}-3074$ Muri b. Bern

gerhard.kocher@muri-be.ch
Die Organisation für wirtschaftliche Zusammenarbeit und Entwicklung OECD in Paris hat wiederum ihre jährliche CD «OECD Health Data 2009» publiziert [1]. Diese 18. Auflage umfasst 1200 Indikatoren mit Zahlenreihen über die Jahre 1960 bis 2006/2007. Die 660000 Daten betreffen Gesundheitszustand, Ressourcen und Inanspruchnahme, Medikamente, Ausgaben und Finanzierung, soziale Sicherheit, nichtmedizinische Gesundheitsfaktoren sowie demografische und wirtschaftliche Entwicklung. Erfasst sind die 30 OECDLänder, wobei aber immer noch zahlreiche Daten einzelner Länder fehlen. $\mathrm{Zu}$ diesen Sündern gehört die Schweiz, die auch für wichtige Statistiken teilweise keine Zahlen liefern kann. Gleichwohl gibt es keine umfangreichere Statistiksammlung über die Gesundheitswesen der 30 Länder. Sie basiert auf einer ausgeklügelten Software, damit die Daten nach Belieben zusammengestellt und zum Beispiel mit Excel weiter ausgewertet werden können.

Im November wird die Ausgabe 2009 des OECDBuches «Health at a glance» erscheinen (französische

\section{Tabelle 1}

Gesamtgesundheitsausgaben Schweiz, 1998-2007. in Millionen Fr.

\begin{tabular}{llll} 
Jahr & Gesamtausgaben & Zunahme (Mio Fr.) & Zunahme in \% \\
\hline 1998 & 39815 & 1605 & 4,2 \\
\hline 1999 & 41104 & 1289 & 3,2 \\
\hline 2000 & 42843 & 1739 & 4,2 \\
\hline 2001 & 45573 & 2730 & 6,4 \\
\hline 2002 & 47389 & 1816 & 4,0 \\
\hline 2003 & 49265 & 1876 & 4,0 \\
\hline 2004 & 51008 & 1743 & 3,5 \\
\hline 2005 & 52043 & 1035 & 2,0 \\
\hline 2006 & 52773 & 730 & 1,4 \\
\hline $2007 *$ & 55336 & 2563 & 4,9 \\
\hline Durchschnitt & & 1713 & 3,8 \\
\hline * Schätzung & & & \\
\hline
\end{tabular}

Ausgabe: «Panorama de la santé»). Die rund 200seitige Übersicht wird zahlreiche Grafiken und Statistiken mit Erläuterungen zu den in der CD behandelten Sachgebieten enthalten. Die letzte Auflage erschien 2007.

\section{Schweizer Gesundheitsausgaben: von Jahr zu Jahr 1,7 Milliarden Franken mehr} Im Schnitt der Jahre 1998 bis 2007 stiegen die Gesamtausgaben für unser Gesundheitswesen um 3,8\% pro Jahr mit einer Schwankungsbreite von 1,4 bis 6,4\% (Tab. 1). Pro Tag wurden 4,7 Millionen Franken mehr ausgegeben als im Vorjahr. 2007 waren es sogar 7 Millionen Franken mehr als 2006. Eine KOF-Prognose [2] schätzt die Gesundheitsausgaben für 2008 auf 58,287 Mrd. Fr., für 2009 auf 60,802 Mrd. und für 2010 auf 62,566 Mrd. Fr. Das jährliche Ausgabenwachstum in diesen drei Jahren soll 4,2\% betragen, die Mehrausgaben pro Jahr werden auf 2,54 Mrd. Fr. geschätzt. Die 60,802 Mrd. Fr. für das laufende Jahr würden 6,9 Millionen Fr. pro Stunde entsprechen.

\section{Gesundheitsausgaben in Prozent des BIP: \\ Schweiz im 3. Rang}

Das Schweizer Gesundheitswesen ist mit Ausgaben in der Höhe von 10,8\% des Bruttoinlandprodukts das drittteuerste der OECD-Länder (Tab. 2). Von 2001 bis 2005 waren wir immer im 2. Rang nach den USA. Seit 2006 sind wir im 3. Rang nach USA und Frankreich. Unser BIP-Anteil 2007 ist 20\% höher als der OECDDurchschnitt, Frankreich liegt 22\% über dem Durchschnitt. Dass das US-Gesundheitssystem ein extremer Sonderfall ist, zeigt sich einmal mehr: Die Gesundheitsausgaben 2007 waren 78\% höher als der OECDDurchschnitt. Nächstes Jahr werden die USA trotz aller Bemühungen der Obama-Regierung ein zweifelhaftes Jubiläum begehen können: Seit 50 Jahren (Beginn der vorliegenden OECD-Statistiken) haben sie die weltweit höchsten Gesundheitsausgaben in Prozent des Bruttoinlandprodukts. Interessanterweise war 1960 aber die Differenz zur Schweiz noch gering: 
Tabelle 2

Gesundheitsausgaben in \% des Bruttoinlandprodukts BIP.

\begin{tabular}{lccc} 
Land & $\mathbf{2 0 0 0}$ & $\mathbf{2 0 0 7}$ & $\begin{array}{c}\text { Zu-/Abnahme } \\
\text { in \% }\end{array}$ \\
\hline USA & 13,6 & 16 & 17,6 \\
\hline Frankreich & 10,1 & 11 & 8,9 \\
\hline Schweiz & $\mathbf{1 0 , 2}$ & $\mathbf{1 0 , 8}$ & 5,9 \\
\hline Deutschland & 10,3 & 10,4 & 1,0 \\
\hline Belgien & 8,6 & 10,2 & 18,6 \\
\hline Kanada & 8,8 & 10,1 & 14,8 \\
\hline Österreich & 9,9 & 10,1 & 2,0 \\
\hline Portugal * & & 9,9 & \\
\hline Dänemark & 8,3 & 9,8 & 18,1 \\
\hline Niederlande & 8 & 9,8 & 22,5 \\
\hline Griechenland & 7,9 & 9,6 & 21,5 \\
\hline Island & 9,5 & 9,3 & $-2,1$ \\
\hline Neuseeland & 7,7 & 9,2 & 19,5 \\
\hline Schweden & 8,2 & 9,1 & 11,0 \\
\hline Norwegen & 8,4 & 8,9 & 6,0 \\
\hline Australien * & 8,3 & 8,7 & 4,8 \\
\hline Italien & 8,1 & 8,7 & 7,4 \\
\hline Spanien & 7,2 & 8,5 & 18,1 \\
\hline Grossbritannien & 7 & 8,4 & 20,0 \\
\hline Finnland & 7,2 & 8,2 & 13,9 \\
\hline Japan * & 7,7 & 8,1 & 5,2 \\
\hline Slowakei & 5,5 & 7,7 & 40,0 \\
\hline Irland & 6,3 & 7,6 & 20,6 \\
\hline Ungarn & 6,9 & 7,4 & 7,2 \\
\hline Luxemburg & 5,8 & 7,3 & 25,9 \\
\hline Südkorea & 4,9 & 6,8 & 38,8 \\
\hline Tschechien & 6,5 & 6,8 & 4,6 \\
\hline Polen & 5,5 & 6,4 & 16,4 \\
\hline Mexiko & 5,1 & 5,9 & 15,7 \\
\hline Durchschnitt & 7,9 & 9,0 & 14,4 \\
\hline * Spalte 2007: Zahl für 2006 & & \\
\hline & & & \\
\hline
\end{tabular}

USA 5,2\%, Schweiz 4,9\% des BIP. Die US-Ausgaben/ BIP waren damit nur $6 \%$ höher als die schweizerischen. Bis 2007 nahm der Unterschied dann auf das Achtfache zu.

Zu grössten Diskussionen und Polemiken führt in den USA immer wieder ein naheliegender Vergleich. Kanada wendet für sein Gesundheitssystem erheblich weniger auf als die Amerikaner: $10,1 \%$ statt $16 \%$ des BIP, also nur $58 \%$. Und dies, obwohl die Systeme qualitativ vergleichbar sind und obwohl in Kanada nicht $16 \%$ der Bevölkerung (47 Millionen Menschen) keine Krankenversicherung haben wie in den USA.

Von 2000 bis 2007 stiegen im OECD-Durchschnitt die Gesundheitsausgaben in Prozent des BIP um 14,4\%. Im Zunahme-Ranking der 28 Länder ist die Schweiz mit 5,9\% im 17. Rang. Die USA sind mit $17,6 \% \mathrm{Zu}-$ nahme im 11. Rang. Trotz ihres im Jahr 2000 bereits weltweit höchsten Ausgabenniveaus verzeichneten die
USA damit bis 2007 wiederum eine überdurchschnittliche Kostenzunahme.

Dass die Gesundheitsausgaben im Verhältnis zum BIP von Jahr zu Jahr zunehmen, ist die Regel. Es gibt aber Ausnahmen. In der Schweiz sind sie selten, und die Reduktion belief sich jeweils nur auf 0,1 oder 0,2 Prozentpunkte. $\mathrm{Zu}$ verzeichnen sind aber auch signifikante Abnahmen. So sank in Schweden der Anteil am BIP zwischen 1982 und 1991 von 9,2 auf $8 \%$ und zwischen 2003 und 2007 von 9,4 auf 9,1 Prozent. Ähnlich war die Entwicklung in Dänemark: von 9,2\% (1982) auf 8,1\% (1995).

Bei allen diesen Vergleichen ist zu beachten, dass es sich um Brüche handelt (Kosten/BIP). Wenn die Gesundheitsausgaben im betreffenden Jahr stärker steigen, das BIP aber stagniert oder sinkt, wird der Prozentanteil kleiner, und umgekehrt. Dieses ungleich starke Wachstum von Zähler und Nenner ist die Regel: von 2000 bis 2007 stiegen in den OECD-Ländern die Gesundheitsausgaben durchschnittlich um 59\%, das BIP nur um $40 \%$.

Wegen der aktuellen Wirtschaftskrise mit geringerem BIP-Wachstum oder auch BIP-Abnahme und unterschiedlichem Kostenwachstum können sich für die Jahre 2008 und vor allem 2009 (und folgende?) grosse Änderungen ergeben, auch in den Ranglisten. Wie jedes Jahr müssen deshalb für internationale Vergleiche auch die Pro-Kopf-Ausgaben (in US-\$) für das Gesundheitswesen herangezogen werden.

\section{Gesundheitsausgaben pro Einwohner: Schweiz zweimal im 3. Rang}

Die Tabelle 3 zeigt wiederum den Sonderfall USA mit kaufkraftbereinigten 7290 \$ pro Kopf (2007). Dies ist 2,4mal mehr als der OECD-Durchschnitt (3065 \$). Norwegen im 2. Rang ist 1,6mal höher als der Durchschnitt, die Schweiz wiederum im 3. Rang 1,4mal höher. Die Pro-Kopf-Ausgaben der USA sind 65\% höher als die schweizerischen. 2007 beliefen sich die US-Gesamtausgaben auf 2,199 Billionen \$ (2199 Milliarden). Dies entspricht einer Zunahme seit $2000 \mathrm{um}$ 65,6\%. Pro Einwohner beträgt die Zunahme 55\%. Für das laufende Jahr schätzt die Regierung die Gesundheitsausgaben auf über 2,5 Billionen (in den USA trillions genannt). Dies entspricht $8160 \$$ pro Einwohner und 17,6\% des Bruttoinlandprodukts.

Nicht kaufkraftbereinigt, also ohne Berücksichtigung der unterschiedlichen Kaufkraft in den verschiedenen Ländern, sieht die Statistik für die USA erheblich besser aus (Tab. 4). Zum Wechselkurs gerechnet liegt 2007 Norwegen mit 7354 \$/Kopf an der Spitze, knapp gefolgt von den USA mit 7290 \$. Die Schweiz ist mit 6108 \$ einmal mehr im 3. Rang. Norwegen liegt $102 \%$ über dem OECD-Durchschnitt von 3646 , die Ausgaben der USA sind $100 \%$ und die der Schweiz $68 \%$ über dem Durchschnitt.

Von 2000 bis 2007 stiegen im OECD-Durchschnitt die Gesundheitsausgaben (ohne Kaufkraftbereinigung) 
Tabelle 3

Gesundheitsausgaben pro Kopf in US-\$ (kaufkraftbereinigt), 2000 und 2007.

\begin{tabular}{lccc} 
Land & 2000 & 2007 & $\begin{array}{l}\text { Zunahme } \\
\text { in \% }\end{array}$ \\
\hline USA & 4704 & 7290 & 55,0 \\
\hline Norwegen & 3039 & 4763 & 56,7 \\
\hline Schweiz * & 3217 & 4417 & 37,3 \\
\hline Luxemburg * & 2553 & 4162 & 63,0 \\
\hline Kanada & 2516 & 3895 & 54,8 \\
\hline Niederlande * & 2337 & 3837 & 64,2 \\
\hline Österreich & 2824 & 3763 & 33,3 \\
\hline Frankreich & 2542 & 3601 & 41,7 \\
\hline Belgien * & 2377 & 3595 & 51,2 \\
\hline Deutschland & 2671 & 3588 & 34,3 \\
\hline Dänemark & 2378 & 3512 & 47,7 \\
\hline Irland & 1805 & 3424 & 89,7 \\
\hline Schweden & 2283 & 3323 & 45,6 \\
\hline Island & 2736 & 3319 & 21,3 \\
\hline Australien * & 2271 & 3137 & 38,1 \\
\hline Grossbritannien & 1833 & 2992 & 63,2 \\
\hline Finnland & 1853 & 2840 & 53,3 \\
\hline Griechenland & 1449 & 2727 & 88,2 \\
\hline Italien & 2052 & 2686 & 30,9 \\
\hline Spanien & 1536 & 2671 & 73,9 \\
\hline Japan * & 1967 & 2581 & 31,2 \\
\hline Neuseeland & 1605 & 2510 & 56,4 \\
\hline Portugal * & 1509 & 2150 & 42,5 \\
\hline Südkorea & 809 & 1688 & 108,7 \\
\hline Tschechien & 980 & 1626 & 65,9 \\
\hline Slowakei & 603 & 1555 & 157,9 \\
\hline Ungarn & 852 & 1388 & 62,9 \\
\hline Polen & 583 & 1035 & 77,5 \\
\hline Mexiko & 508 & 823 & 62,0 \\
\hline Durchschnitt & 2014 & 3065 & 58,9 \\
\hline * Spalte 2007: Zahl für 2006 & & \\
\hline & & & \\
\hline
\end{tabular}

auf mehr als das Doppelte, nämlich um 106\%. In dieser Rangliste sind die Schweiz und die USA ganz unten zu finden. Sie belegen die Ränge 26 und 28 von 29 Ländern.

\section{Ausgaben für Prävention und öffentliche Gesundheit: Schweiz abnehmend}

Gemessen an den nationalen Gesundheitsausgaben gibt Kanada am meisten für Prävention und öffentliche Gesundheit aus: 2007 waren es 6,9\%. Finnland folgt mit 5,4\%, Neuseeland mit 4,9\% (Tab. 5, linke Hälfte). Die Schweiz erreichte mit 2,3\% einen mittleren Rang. Der Durchschnitt der erfassten 24 Länder beträgt 3\% gegenüber 2,5\% im Jahr 2000. Der Prävention und Public Health wird in Wissenschaft, Medien und Politik (zumindest rhetorisch) grosse Bedeutung zugemessen. Da überrascht es, dass seit 2000 dieser Ausgabenanteil nur in 9 Ländern gestiegen, in einem

\section{Tabelle 4}

Gesundheitsausgaben pro Kopf in US-\$ (zu Wechselkursen), 2000 und 2007.

\begin{tabular}{|c|c|c|c|}
\hline Land & 2000 & 2007 & $\begin{array}{l}\text { Zu-/Abnahme } \\
\text { in } \%\end{array}$ \\
\hline Norwegen & 3156 & 7354 & 133,0 \\
\hline USA & 4704 & 7290 & 55,0 \\
\hline Schweiz & 3529 & 6108 & 73,1 \\
\hline Island & 2938 & 5964 & 103,0 \\
\hline Dänemark & 2479 & 5550 & 123,9 \\
\hline Luxemburg * & 2205 & 4929 & 123,5 \\
\hline Niederlande & 1916 & 4640 & 142,2 \\
\hline Irland & 1595 & 4556 & 185,6 \\
\hline Österreich & 2335 & 4523 & 93,7 \\
\hline Schweden & 2280 & 4495 & 97,1 \\
\hline Frankreich & 2192 & 4491 & 104,9 \\
\hline Kanada & 2082 & 4403 & 111,5 \\
\hline Belgien & 1945 & 4389 & 125,7 \\
\hline Deutschland & 2372 & 4209 & 77,4 \\
\hline Grossbritannien & 1769 & 3867 & 118,6 \\
\hline Finnland & 1693 & 3809 & 125,0 \\
\hline Australien * & 1734 & 3321 & 91,5 \\
\hline Italien & 1541 & 3136 & 103,5 \\
\hline Neuseeland & 1055 & 2855 & 170,6 \\
\hline Japan * & 2827 & 2759 & $-2,4$ \\
\hline Spanien & 1036 & 2712 & 161,8 \\
\hline Griechenland & 919 & 2679 & 191,5 \\
\hline Portugal * & 970 & 1823 & 87,9 \\
\hline Südkorea & 536 & 1362 & 154,1 \\
\hline Tschechien & 361 & 1141 & 216,1 \\
\hline Slowakei & 208 & 1077 & 417,8 \\
\hline Ungarn & 326 & 1019 & 212,6 \\
\hline Polen & 247 & 716 & 189,9 \\
\hline Mexiko & 328 & 567 & 72,9 \\
\hline Durchschnitt & 1768 & 3646 & 133,1 \\
\hline
\end{tabular}

Land gleichgeblieben und in 7 Ländern sogar gesunken ist. Dazu gehört auch die Schweiz mit einem Rückgang von 4,2\%. «Die Ausgaben für Leistungen im Bereich der Prävention und Gesundheitsförderung sind im Vergleich zu den Ausgaben für die Kuration verschwindend klein», kritisieren Stefan Spycher, Hélène Jaccard Ruedin, Gabriele Wiedenmayer und Christoph Junker im Nationalen Gesundheitsbericht 2008 [3]. Unter vielen anderen weisen auch die OECDund WHO-Experten in ihrem Bericht [4] auf das «herrschende Ungleichgewicht zwischen Prävention und kurativer Medizin» in der Schweiz hin.

Die rechte Hälfte der Tabelle 5 zeigt den Anteil der öffentlichen Ausgaben für Prävention und öffentliche Gesundheit an den Gesamt-Gesundheitsausgaben der einzelnen Länder. Er beträgt im Durchschnitt 2,4\%. Die Schweiz erreicht mit ihren 1,5\% (unverändert gegenüber 2000) nur den 20. Rang der 24 Länder. 
Tabelle 5

Ausgaben für Prävention und öffentliche Gesundheit, 2000 und 2007.

Gesamtausgaben für Prävention und öffentliche Gesundheit in \% der Gesamtgesundheitsausgaben

\begin{tabular}{|c|c|c|c|c|c|c|}
\hline Land & 2000 & 2007 & $\begin{array}{l}\text { Zu-/Abnahme } \\
\text { in } \%\end{array}$ & 2000 & 2007 & $\begin{array}{l}\text { Zu-/Abnahme } \\
\text { in \% }\end{array}$ \\
\hline Kanada & 5,9 & 6,9 & 16,9 & 5,9 & 6,9 & 16,9 \\
\hline Finnland & 4,6 & 5,4 & 17,4 & 2,8 & 3,5 & 25,0 \\
\hline Neuseeland & & 4,9 & & & 4,6 & \\
\hline Niederlande & 5,1 & 4,7 & $-7,8$ & 2,4 & 2,4 & 0,0 \\
\hline Slowakei & & 4,7 & & 0 & 2 & \\
\hline Ungarn & 4,9 & 4 & $-18,4$ & 2,4 & 2,6 & 8,3 \\
\hline Belgien & & 3,9 & & & 3,9 & \\
\hline Deutschland & 3,1 & 3,5 & 12,9 & 2,6 & 3 & 15,4 \\
\hline Schweden & & 3,5 & & & 2,6 & \\
\hline USA & 3,9 & 3,1 & $-20,5$ & 3,5 & 3,1 & $-11,4$ \\
\hline Mexiko & 0,6 & 2,8 & 366,7 & 0,6 & 2,8 & 366,7 \\
\hline Schweiz & 2,4 & 2,3 & $-4,2$ & 1,5 & 1,5 & 0,0 \\
\hline Spanien & 1,1 & 2,3 & 109,1 & 1,1 & 2,2 & 100,0 \\
\hline Polen & & 2,2 & & & 1,6 & \\
\hline Tschechien & 1,5 & 2,2 & 46,7 & 1,5 & 1,8 & 20,0 \\
\hline Frankreich & 2,2 & 1,9 & $-13,6$ & 1,5 & 1,3 & $-13,3$ \\
\hline Südkorea & 0,7 & 1,9 & 171,4 & 0,6 & 1,8 & 200,0 \\
\hline Norwegen & & 1,9 & & & 1,7 & \\
\hline Portugal * & 2,4 & 1,8 & $-25,0$ & 1,4 & 1,1 & $-21,4$ \\
\hline Österreich & 1,5 & 1,8 & 20,0 & 1,3 & 1,7 & 30,8 \\
\hline Australien * & 1,9 & 1,6 & $-15,8$ & 1,9 & 1,6 & $-15,8$ \\
\hline Island & 0,5 & 1,6 & 220,0 & 0,5 & 1,6 & 220,0 \\
\hline Dänemark & & 1,4 & & & 1,3 & \\
\hline Italien & 0,6 & 0,6 & 0,0 & 0,6 & 0,6 & 0,0 \\
\hline Durchschnitt & 2,5 & 3,0 & & 1,8 & 2,4 & \\
\hline
\end{tabular}

Zahl der Arztbesuche in der Schweiz sehr tief Dass die Bevölkerung «wegen jedem Bobo zum Arzt geht», ist ein oft gehörter Vorwurf von Politikern, Kassenvertretern und anderen Personen. Gemäss einer repräsentativen Umfrage sieht die Bevölkerung dies bei weitem als die wichtigste Ursache der Prämiensteigerungen an (Sondage Santé 2009, santésuisse). 9 von 10 Patienten gehen allerdings berechtigterweise zu einem Arzt, meint dagegen Heinz Zimmermann, Chefarzt der Notfallstation des Berner Inselspitals. Gemäss den OECD-Zahlen (Tab. 6) ist die Häufigkeit der Arztbesuche in der Schweiz tatsächlich tief: 4 pro Jahr (2007). Dies ist $41 \%$ unter dem Durchschnitt der verglichenen OECD-Länder. Die Schweiz ist damit im 21. Rang der 23 Länder. Eine Erhebung der santésuisse zur obligatorischen Krankenversicherung kam 2006 auf 5,5 Konsultationen pro Jahr. Dabei zeigten sich grosse kantonale Unterschiede: von 3,9 Arztbesuchen im Jura bis zu 6,2 im Kanton Basel-Stadt.

\section{Hohe Akutbettenbelegung in der Schweiz}

Zum Thema Krankenhaus enthielt der letztjährige SÄZ-Artikel [5] Statistiken für 2006 zur Bettendichte und zur Zahl der Krankenhaustage in der Akutversorgung. Mit 3,5 Spitalbetten pro 1000 Einwohner war die Schweiz im 20. Rang von 25 Ländern und weit unter dem Durchschnitt von 5,5 Betten. Für 2007 ist die Schweizer Bettendichte unverändert. Bei den Krankenhaustagen pro Einwohner und Jahr lag die Schweiz genau im Schnitt der erfassten Länder (1,1 Tage). Auch dieser Wert blieb 2007 gleich.

Der vorliegende Artikel zeigt erstmals die Bettenbelegung in der Akutversorgung (Tab. 7). In der Schweiz ist sie erheblich höher als im Durchschnitt der erfassten 18 OECD-Länder, nämlich 85,2\% gegenüber 76,5\%. Damit ist die Schweiz im 4. Rang. Japan, Deutschland und Frankreich sind nahe beim Durchschnittswert. In den USA dagegen ist die Zahl der «kalten Betten» auffallend hoch, nämlich ein Drittel. Dies ist mehr als doppelt so hoch wie bei uns. Diese schlechte Auslastung in den USA hängt unter anderem mit der geringen Zahl der Krankenhaustage pro Einwohner und Jahr zusammen: 2006 waren es 0,7 Tage, 2007 sogar nur noch 0,6 Tage gegenüber durchschnittlich 1,1 Tagen in den erfassten OECD-Ländern und auch in der Schweiz. 
Tabelle 6

Arztbesuche pro Kopf und Jahr, 2000 und 2007.

\begin{tabular}{|c|c|c|c|}
\hline Land & 2000 & $2007^{*}$ & $\begin{array}{l}\text { Zu-/Abnahme } \\
\text { in \% }\end{array}$ \\
\hline Japan & 14,4 & 13,6 & $-5,6$ \\
\hline Tschechien & 12,6 & 12,6 & 0,0 \\
\hline Slowakei & 15 & 11,2 & $-25,3$ \\
\hline Ungarn & 11,1 & 10,8 & $-2,7$ \\
\hline Spanien & & 8,1 & \\
\hline Belgien & 7,9 & 7,6 & $-3,8$ \\
\hline Deutschland & 7,3 & 7,5 & 2,7 \\
\hline Polen & 5,4 & 6,8 & 25,9 \\
\hline Österreich & 6,7 & 6,7 & 0,0 \\
\hline Island & 5,8 & 6,5 & 12,1 \\
\hline Australien & 6,4 & 6,3 & $-1,6$ \\
\hline Frankreich & 6,9 & 6,3 & $-8,7$ \\
\hline Luxemburg & 6,1 & 6,1 & 0,0 \\
\hline Kanada & 6,3 & 5,8 & $-7,9$ \\
\hline Niederlande & 5,9 & 5,7 & $-3,4$ \\
\hline Türkei & 2,5 & 5,6 & 124,0 \\
\hline Grossbritannien & 5,3 & 5 & $-5,7$ \\
\hline Neuseeland & & 4,7 & \\
\hline Finnland & 4,3 & 4,2 & $-2,3$ \\
\hline Portugal & 3,5 & 4,1 & 17,1 \\
\hline Schweiz & & 4 & \\
\hline USA & 3,7 & 3,8 & 2,7 \\
\hline Schweden & 2,8 & 2,8 & 0,0 \\
\hline Durchschnitt & 7,0 & 6,8 & 5,9 \\
\hline
\end{tabular}

Tabelle 7

Akutbettenbelegung in \%, 2000 und 2007

\begin{tabular}{llll} 
Land & $\mathbf{2 0 0 0}$ & $\mathbf{2 0 0 7}$ & $\begin{array}{l}\text { Zu-/Abnahme } \\
\text { in \% }\end{array}$ \\
\hline Kanada* & 91,2 & 89 & $-2,4$ \\
\hline Norwegen & 85,2 & 88 & 3,3 \\
\hline Irland* & 84,5 & 86,7 & 2,6 \\
\hline Schweiz & $\mathbf{8 4 , 8}$ & $\mathbf{8 5 , 2}$ & $\mathbf{0 , 5}$ \\
\hline Grossbritannien & 82,2 & 83,3 & 1,3 \\
\hline Österreich & 77,3 & 79,4 & 2,7 \\
\hline Italien* & 75,6 & 78,4 & 3,7 \\
\hline Spanien* & 77,1 & 78,2 & 1,4 \\
\hline Japan & 81,8 & 76,4 & $-6,6$ \\
\hline Deutschland & 81,1 & 76 & $-6,3$ \\
\hline Frankreich & 75 & 74 & $-1,3$ \\
\hline Australien * & 70,4 & 73,5 & 4,4 \\
\hline Portugal & 71,3 & 72,6 & 1,8 \\
\hline Tschechien & 70,5 & 72,2 & 2,4 \\
\hline Ungarn & 73,2 & 69,2 & $-5,5$ \\
\hline Slowakei & 70,6 & 67,9 & $-3,8$ \\
\hline USA & 63,9 & 66,6 & 4,2 \\
\hline Mexiko & 57,4 & 59,6 & 3,8 \\
\hline Durchschnitt & 76,3 & 76,5 & 0,4 \\
\hline * Spalte 2007: Zahl für 2006 & & \\
\hline & & & \\
\hline & & & \\
\hline
\end{tabular}

\section{Ausstattung mit medizintechnischen Geräten}

Die Bedeutung der Medizintechnik steigt ständig - in Diagnostik, Therapie und Rehabilitation, für Kosten und Erträge sowie für die Volkswirtschaft (Arbeitsplätze und Exporte). Die Ausgaben für Medizintechnik machen mindestens 5\% der nationalen Gesundheitsausgaben aus, 2009 also mindestens 3 Milliarden Franken [6]. Dazu kommen die erheblichen indirekten Ausgaben und die Folgekosten. Leider ist die Datenlage zur Medizintechnik immer noch dürftig, auch in anderen Ländern.

Die CD enthält immerhin Daten zu fünf Typen von medizintechnischen Geräten, wenn auch nur für 19 bis 24 der 30 OECD-Länder (Tab. 8). Bezüglich Gerätedichte pro Million Einwohner 2007 ist die Schweiz im 3. Rang bei den Lithotriptern, im 4. Rang bei Strahlentherapiegeräten, im 6. Rang bei den Magnetresonanz-Tomographen und im 9. Rang bei den Computertomographen. Für die Mammographen lieferte die Schweiz der OECD keine Daten. Die Mammographendichte der erfassten 18 Länder beträgt 19,9 pro Million Einwohner. Dieser Dichte entsprächen für die Schweiz 153 Mammographen.

Zwischen den Kantonen variiert übrigens die Gerätedichte je nach Gerät zwischen 1:2,9 bis 1:7,2. $\mathrm{Zu}$ beachten ist dabei, dass Appenzell-Innerrhoden weder einen CT noch einen Mammographen hat. Obwalden hat keinen CT und Uri keinen Mammographen.

Zwischen 2000 und 2007 nahm die Gerätedichte in der Schweiz bei den Lithotriptern um 9\% zu, bei den MRI um 12\% und bei den CT um 1\%. Die Gerätedichte in der Strahlentherapie nahm um 6\% ab.

Die OECD-CD enthält auch Angaben über die Anzahl Untersuchungen je Gerät und Jahr. Pro MRI werden im Schnitt von 12 Ländern 4734 Aufnahmen gemacht (Extreme: USA 3521, Ungarn 10029). Die Zahl der Untersuchungen pro Computertomograph variiert zwischen 2576 in Deutschland und 8624 in Kanada. Der Durchschnitt der erfassten 11 Länder beträgt 5760 .

\section{Erhebliche Diskrepanzen zwischen vergleichbaren Ländern}

Diese und zahlreiche andere Dichtezahlen zeigen deutlich die grossen Unterschiede zwischen vergleichbaren Ländern. Die alte Erkenntnis «Alle Medizin ist lokal» kann auf anderen Gebieten durch «Alle Medizin ist national» ergänzt werden. Interessant und mit feinem Humor beschrieben hat dies Lynn Payer 1993 in ihrem Bestseller «Andere Länder, andere Leiden Ärzte und Patienten in England, Frankreich, den USA und hierzulande [Deutschland]» [7].

Die OECD-CD ist ebenfalls voller Belege dafür, dass zwischen Gesundheitssystemen, die auf den ersten Blick ähnlich erscheinen, eklatante Pro-KopfDifferenzen bezüglich Zahl der Einrichtungen, Inanspruchnahme medizinischer Leistungen, Kosten und Finanzierung bestehen. Diese Erkenntnis sollte dazu führen, den Status quo im eigenen Land nicht unbesehen als richtig und «normal» anzusehen, sondern 
auf Unter-, Über- und Fehlversorgung abzuklopfen. Und natürlich auch, dafür zu sorgen, dass die nach 18 Jahren OECD-CDs noch immer fehlenden Schweizer Daten erhoben werden, damit unser Gesundheitssystem mit dem anderer Länder verglichen werden kann. Der Worte sind genug gewechselt, nun lasst uns endlich Daten sehen ...

\section{Literatur/Anmerkungen}

1 OECD Health Data 2009. Statistics and Indicators for 30 countries. Paris: OECD Publishing; 2009. CD-ROM englisch/französisch/deutsch/spanisch mit Benutzerhandbuch, $80 €$. www.oecd.org/health/healthdata.

2 Konjunkturforschungsstelle ETH Zürich (KOF), Mai 2009. - S. auch: M. Vuilleumier et al. Déterminants et évolution des coûts du système de santé en Suisse. Revue de la littérature et projections à l'horizon 2030. Neuchâtel: Office fédéral de la statistique, 2007.
3 Gesundheit in der Schweiz - Nationaler Gesundheitsbericht 2008. Hg. Katharina Meyer, Verlag Hans Huber, Bern 2009. Volltext: www.obsan.admin.ch

4 Schweiz. OECD-Berichte über Gesundheitssysteme. Paris: OECD, 2006. Auch in Französisch und Englisch erschienen.

5 Schweiz Ärztezeitung. 2008:89:44.

6 s. dazu: Medical Cluster Booklet 2009. Bern: Medical Cluster, o. J. - Swiss Medtech Report 2008. O. V., o. O., o. J. - The Swiss Medical Technology Industry 2008 Survey. o. O., Medical Cluster/Helbling/Roland Berger, 2008. - Gerhard Kocher: Medizintechnik, in: Gesundheitswesen Schweiz 2007-2009 Eine aktuelle Übersicht. Bern: Hans Huber, 2007.

7 Frankfurt: Campus; Original: «Medicine and Culture. Varieties of Treatment in the United States, England, West Germany, and France», New York 1988, 2. überarb. Auflage 1996.

\section{Tabelle 8}

Medizintechnische Geräte pro Million Einwohner, 2000 und 2007.

\begin{tabular}{|c|c|c|c|c|c|c|c|c|c|c|}
\hline Land & $\begin{array}{l}\text { CT } \\
2000\end{array}$ & $\begin{array}{l}\text { CT } \\
2007\end{array}$ & $\begin{array}{l}\text { MRT } \\
2000\end{array}$ & $\begin{array}{l}\text { MRT } \\
2007\end{array}$ & $\begin{array}{l}\text { Str } \\
2000\end{array}$ & $\begin{array}{l}\text { Str } \\
2007\end{array}$ & $\begin{array}{l}\text { Lith } \\
2000\end{array}$ & $\begin{array}{l}\text { Lith } \\
2007\end{array}$ & $\begin{array}{l}\text { Mam } \\
2000\end{array}$ & $\begin{array}{l}\text { Mam } \\
2007\end{array}$ \\
\hline Australien & 26,1 & $\ldots$ & 3,5 & 5,1 & 5,2 & $\ldots$ & 1,3 & $\ldots$ & $\ldots$ & 24,2 \\
\hline Belgien & 21,8 & 41,6 & 6 & 7,5 & 12,5 & 14,1 & 4,8 & 4,6 & $\ldots$ & 19,6 \\
\hline Dänemark & 11,4 & 17,4 & 5,4 & $\ldots$ & 5,4 & 8,2 & $\ldots$ & $\ldots$ & $\ldots$ & 10,4 \\
\hline Deutschland & 12,7 & 16,3 & 4,9 & 8,2 & 4,8 & 4,7 & 3 & 3,9 & $\ldots$ & $\ldots$ \\
\hline Finnland & 13,5 & 16,4 & 9,9 & 15,3 & 8,7 & 8,7 & 0,4 & 0,6 & $\ldots$ & 34,8 \\
\hline Frankreich & 9,5 & 10,3 & 2,6 & 5,7 & 6,1 & 9,1 & 0,9 & 1,5 & 42,5 & $\ldots$ \\
\hline Grossbritannien * & 4,5 & 7,6 & 4,7 & 8,2 & $\ldots$ & 4 & $\ldots$ & $\ldots$ & 6,1 & 8,4 \\
\hline Irland & $\ldots$ & 14,3 & $\ldots$ & 8,5 & $\ldots$ & 8,8 & $\ldots$ & 1,2 & $\ldots$ & 14,3 \\
\hline Island & 21,3 & 32,1 & 10,7 & 19,3 & 14,2 & 12,8 & 3,6 & 3,2 & 17,8 & 16,1 \\
\hline Italien & 21 & 30,3 & 7,7 & 18,6 & 3,7 & 5,4 & $\ldots$ & $\ldots$ & $\ldots$ & 26,9 \\
\hline Kanada & $\ldots$ & 12 & 2,5 & 6,7 & $\ldots$ & $\ldots$ & $\ldots$ & 0,6 & $\ldots$ & $\ldots$ \\
\hline Luxemburg & 25,2 & 27,3 & 2,3 & 10,5 & 4,6 & 4,2 & 2,3 & 2,1 & 22,9 & 23,1 \\
\hline Mexiko & $\ldots$ & 4 & 0,6 & 1,5 & 1,1 & 1,9 & 0,4 & 1,1 & $\ldots$ & 5,2 \\
\hline Neuseeland & 8,8 & 12,3 & $\ldots$ & 8,8 & 9,9 & $\ldots$ & $\ldots$ & 0,5 & $\ldots$ & 27,7 \\
\hline Österreich & 25,8 & 29,8 & 10,9 & 17,7 & 4,2 & 4,8 & 1,7 & 1,9 & $\ldots$ & $\ldots$ \\
\hline Polen & 4,4 & 9,7 & $\ldots$ & 2,7 & $\ldots$ & 9 & $\ldots$ & 3,9 & 11 & 16,5 \\
\hline Portugal & $\ldots$ & 26 & $\ldots$ & 8,9 & $\ldots$ & 10 & $\ldots$ & 3 & $\ldots$ & 35,5 \\
\hline Schweiz & 18,5 & 18,7 & 12,9 & 14,4 & 10,4 & 9,8 & 4,5 & 4,9 & $\ldots$ & $\ldots$ \\
\hline Slowakei & $\ldots$ & 13,7 & $\ldots$ & 5,7 & $\ldots$ & 12,8 & $\ldots$ & 5,7 & $\ldots$ & 14,4 \\
\hline Spanien & 12 & 14,6 & 4,8 & 9,3 & 3,7 & 4,5 & 1,8 & 2 & $\ldots$ & 10,7 \\
\hline Südkorea & 28,4 & 37,1 & 5,4 & 16 & 5,3 & 5,1 & 4,4 & 12,6 & 13,3 & 41,9 \\
\hline Tschechien & 9,6 & 13,1 & 1,7 & 4,4 & 8,5 & 8,8 & 2,8 & 3,2 & 10,6 & 13,5 \\
\hline Türkei & $\ldots$ & 8,1 & $\ldots$ & 5,6 & ... & $\ldots$ & & ... & $\ldots$ & $\ldots$ \\
\hline Ungarn & 5,7 & 7,3 & 1,8 & 2,8 & 2,3 & 3,4 & 0,9 & 1,6 & 9,4 & 14,1 \\
\hline USA & $\ldots$ & 34,3 & 10,9 & 25,9 & $\ldots$ & $\ldots$ & $\ldots$ & $\ldots$ & $\ldots$ & $\ldots$ \\
\hline Durchschnitt & 15,6 & 18,9 & 5,7 & 9,9 & 6,5 & 7,5 & 2,3 & 3,1 & 16,7 & 19,9 \\
\hline Rang der Schweiz & 8 & 9 & 1 & 6 & 3 & 4 & 2 & 3 & $\ldots$ & $\ldots$ \\
\hline Gerätezahl Schweiz & 133 & 141 & 93 & 109 & 75 & 74 & 32 & 37 & $\ldots$ & $\ldots$ \\
\hline
\end{tabular}

CT Computertomographen, MRT Magnetresonanz-Tomographen, Str Strahlentherapiegeräte, Lith Lithotripter, Mam Mammographen * geschätzt. 2007: bei einigen Werten 2006 